Communications in Physics, Vol. 23, No. 1 (2013), pp. 49-55

\title{
MOTT TRANSITION OF THE HALF-FILLED HUBBARD MODEL IN A TWO-DIMENSIONAL FRUSTRATED LATTICE
}

\author{
LE DUC ANH \\ Department of Physics, Hanoi National University of Education \\ HOANG ANH TUAN \\ Institute of Physics, VAST
}

\begin{abstract}
Using coherent potential approximation we study zero-temperature Mott transition of the half-filled Hubbard model in a two-dimensional square lattice with geometrical frustration. It turns out that the geometrical frustration reduces the gap between the Hubbard bands. As a result the metallic phase is stabilized up to a fairly large value of the on-site Coulomb interaction. We found that the critical value $U_{C}$ for the Mott transition is enhanced by the geometrical frustration. Our results are in good agreement with the ones obtained by the single-site dynamical mean-field theory.
\end{abstract}

\section{INTRODUCTION}

Strongly correlated electron system with geometrical frustration exhibits a variety of phenomena and is presently a major topic of great interest in the condensed-matter community $[1,2]$. The competition between strong electronic correlations and the geometrical frustration in metallic spinel compounds may cause some novel phenomena such as heavy fermion state in $\mathrm{LiV}_{2} \mathrm{O}_{4}$ [3], superconductivity with relatively high transition temperature of $T_{C}=13.7 \mathrm{~K}$ in $\mathrm{LiTi}_{2} \mathrm{O}_{4}[4]$, and so forth. New aspects of the Mott metal-insulator transitions are also uncovered by the geometrical frustration, which is now one of the central issues in the physics of strongly correlated electron systems[5]. Among all possible microscopic models, the one-band Hubbard model is often used for a study of the interplay between the geometrical frustration and strong electronic correlations. Previous studies of such model on triangular and Kagomé lattices have been performed by various approaches such as the fluctuation exchange approximation[6], quantum Monte Carlo calculations[7], coherent potential approximation[8], and cellular dynamical mean field theory[9]. A similar problem for other frustrated lattices has been also carried out by means of fourth order perturbative calculation[10], path-integral renormalization group[11], variational Monte Carlo simulations[12], and a cluster extension of dynamical mean-field theory[13].

In this paper, we investigate the Mott transition of the one-band half-filled Hubbard model in a two-dimensional square lattice with geometrical frustration. In order to carry out the calculation we treat the model within the coherent potential approximation (CPA)[14]. Assuming a paramagnetic groundstate, which is valid for the strongly frustrated case, we show that the frustrated system undergoes a metal-insulator transition at a certain critical value of the onsite Coulomb repulsion $U_{C}$. We show that the geometrical frustration 
reduces the gap between the Hubbard bands. As a result the metallic phase is stabilized up to fairly large Hubbard interactions for systems under strong geometrical frustration. This paper is organized as follows. The Hubbard model and the coherent potential approximation are presented in the next section. In Section 3 we discuss our numerical results. Finally, the paper is concluded and the directions of future work are in Section 4.

\section{MODEL AND FORMALISM}

The one-band Hubbard model on the two-dimensional square lattice with nearest hopping $t_{i, j}$ and on-site Hubbard repulsion $U$ reads

$$
H=-\sum_{<i, j>\sigma} t_{i, j}\left(c_{i \sigma}^{+} c_{j \sigma}+c_{j \sigma}^{+} c_{i \sigma}\right)+U \sum_{i} n_{i \uparrow} n_{i \downarrow}-\mu \sum_{i} n_{i}
$$

where $c_{i \sigma}\left(c_{i \sigma}^{+}\right)$annihilates (creates) an electron with spin $\sigma$ at site $i, n_{i \sigma}=c_{i \sigma}^{+} c_{i \sigma}$ and $n_{i}=n_{i \uparrow}+n_{i \downarrow}$. The nearest neighbor hopping parameter $t_{i, j}$, as depicted in Fig. 1 , takes either $t$ or $t^{\prime}$. By introducing $t^{\prime}$, the so-called crossing hopping parameter, one controls the geometrical frustration of the frustrated lattice and clarify how the lattice geometry affects physical properties. The chemical potential $\mu$ is chosen such that the average occupancy is 1 (half-filling).

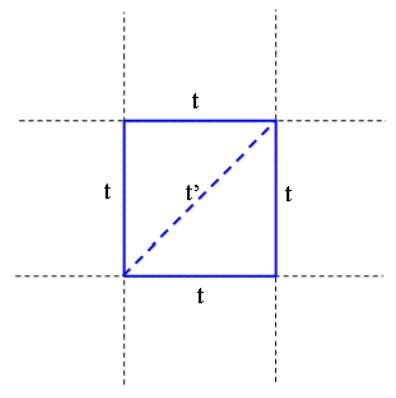

Fig. 1. Hoppings in a two-dimensional frustrated lattice.

In the alloy-analogue approach the many-body Hamiltonian (1) is replaced by a oneparticle Hamiltonian with disorder which is of the form

$$
H=\sum_{i, \sigma} E_{\sigma} n_{i \sigma}-\sum_{<i, j>\sigma} t_{i, j}\left(c_{i \sigma}^{+} c_{j \sigma}+c_{j \sigma}^{+} c_{i \sigma}\right)
$$

where

$$
E_{\sigma}=\left\{\begin{array}{lr}
\mu & \text { with probability } 1-n_{-\sigma}, \\
\mu+U & \text { with probability } n_{-\sigma} .
\end{array}\right.
$$

The Green function of the Hamiltonian (2) has to be averaged over all possible configurations of the random potential, which can be considered to be due to alloy constituents. The averaging cannot be performed exactly. Within the CPA, the Green function is determined by the conditional Green function as follows

$$
G_{\sigma}(\omega)=\frac{F_{\sigma}(\omega)\left(1-n_{-\sigma}\right)}{1+F_{\sigma}(\omega)\left(\Xi_{\sigma}(\omega)+\mu\right)}+\frac{F_{\sigma}(\omega) n_{-\sigma}}{1+F_{\sigma}(\omega)\left(\Xi_{\sigma}(\omega)+\mu-U\right)} .
$$


Here $F_{\sigma}(\omega)$ is the local lattice Green function

$$
F_{\sigma}(\omega)=\int_{-\infty}^{\infty} \frac{\rho_{0}(\varepsilon) d \varepsilon}{\omega+i \eta+\mu-\Xi_{\sigma}(\omega)-\varepsilon}
$$

where the bare density of states of the frustrated lattice $\rho_{0}(\varepsilon)=\frac{1}{4 \pi^{2}} \int_{-\pi}^{\pi} d k_{x} \int_{\pi}^{\pi} d k_{y} \delta\left(\varepsilon-\varepsilon_{\vec{k}}\right)$ and the bare one electron dispersion[13] $\varepsilon_{\vec{k}}=-2 t\left(\cos k_{x}+\cos k_{y}\right)-2 t^{\prime} \cos \left(k_{x}+k_{y}\right)$. The self-consistent condition of the CPA requires that the conditional Green function must coincide with the local Green function of the original lattice, i.e.,

$$
G_{\sigma}(\omega)=F_{\sigma}(\omega)
$$

So far, for determining the Green function we have obtained a closed system of equations, which can be solved numerically by iterations $[15,16]$.

\section{NUMERICAL RESULTS AND DISCUSSION}

We solve numerically the self-consistent equations (4) - (6) to determine the selfenergy and the Green function by simple iterations[15, 16]. The algorithm is summarized as follows. Begin with an initial self-energy guess, one obtains the local Green function from Eq. (5). Substituting the self-energy and the local Green function were calculated in the previous step to Eq. (4) one calculates the conditional Green function. Finally, a new self energy is determined by

$$
\Xi_{\sigma}^{\prime}(\omega)=\Xi_{\sigma}(\omega)+\frac{1}{F_{\sigma}(\omega)}-\frac{1}{G_{\sigma}(\omega)}
$$

which is equivalent to Eq. (6). This procedure is iterated until convergence is reached. Normally, a relative error for the Green function of less than $10^{-10}$ is achieved after few hundreds of iterations. However, the number of iterations for such relative error will be of the order of thousands when one reaches the Mott transition. Throughout this work, for simplicity we assume a paramagnetic solution for the groundstate. Note that for highly frustrated systems at half-filling, this assumption is valid since the antiferromagnetic order is expected to be destroyed by frustration[13, 17]. Hereafter, we take $t=1$ as the energy unit, total band-filling 1 , zero temperature, and $\eta=0.01$ in numerical calculations. Now we turn to present our numerical results for all the unfrustrated model $t^{\prime}=0$, moderately frustrated model $t^{\prime}=0.5$, and strongly frustrated one $t^{\prime}=1$. Fig. 2 shows the non-interacting Green function for various values of the crossing hopping parameter $t^{\prime}$. It is clear that the geometrical frustration, due to the presence of the crossing hopping parameter $t^{\prime}$, makes the non-interacting DOS being asymmetric. The absence of particle-hole symmetry suggests that a system magnetic long-range order should be suppressed, thus, the ground state is likely to be paramagnetic. Furthermore, the absence of particlehole symmetry also implies that for strongly frustrated systems the weights of the Hubbard bands may be different. The latter signifies that the chemical potential for large $U$ at half-filling may lie at one of these Hubbard bands 
or that the Mott insulator may not be formed. However, it is not the case though.

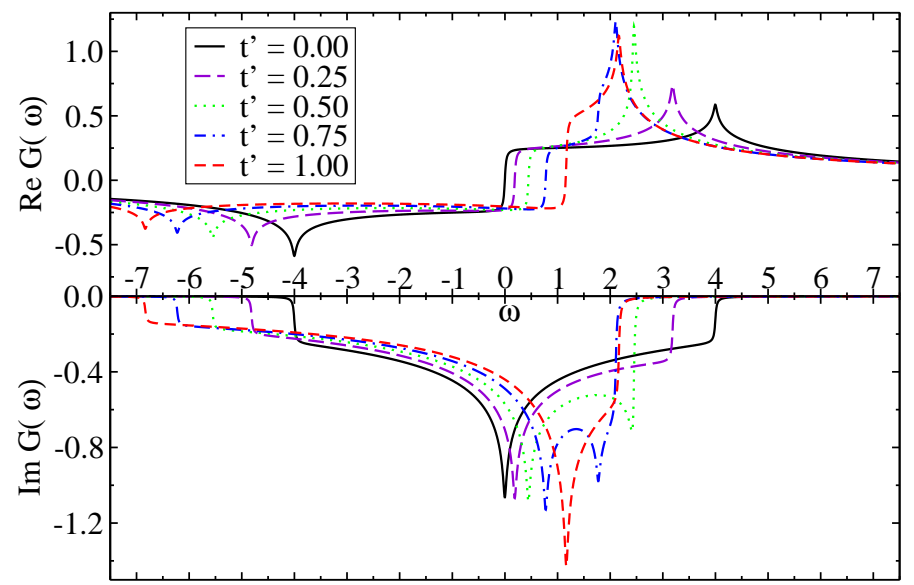

Fig. 2. Non-interacting Green function for various values of the crossing hopping parameter $t^{\prime}$.
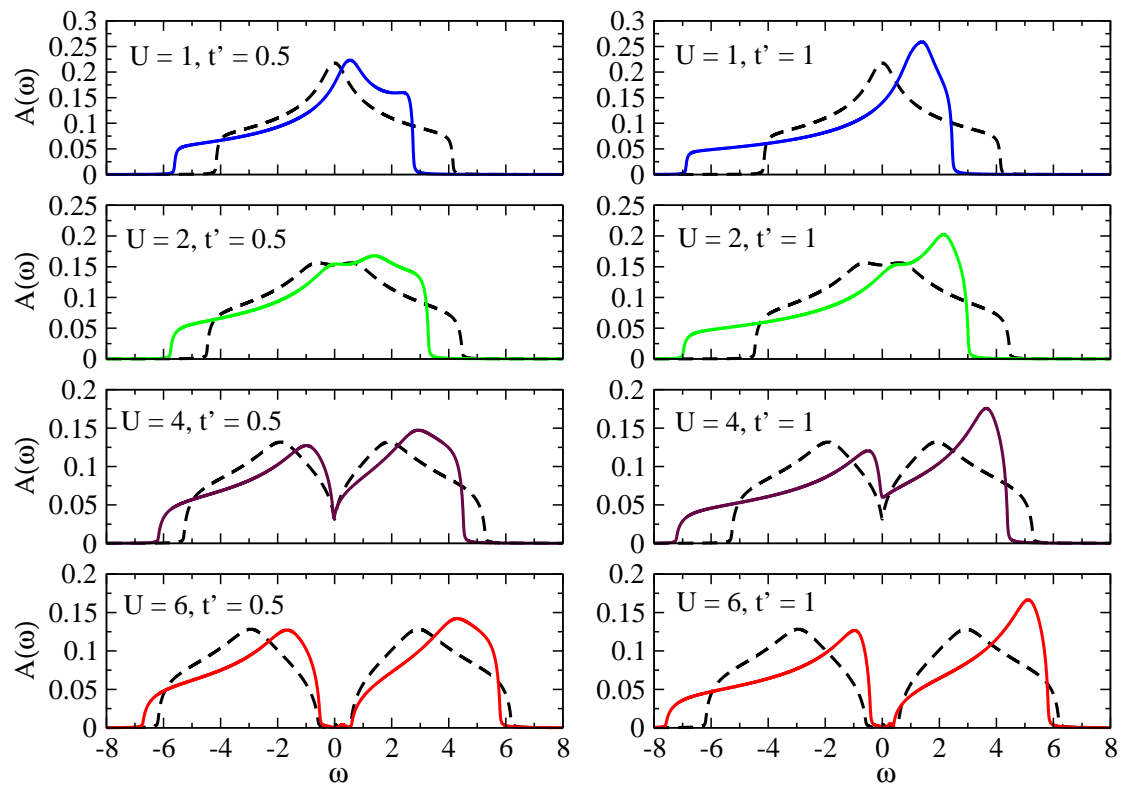

Fig. 3. Spectral functions for the unfrustrated $t=0^{\prime}$ model (dashed lines), moderately frustrated model $t^{\prime}=0.5$ (solid lines on the left panel), and strongly frustrated one $t^{\prime}=1$ (solid lines on the right panel).

From Fig. 3, spectral functions for various values of the onsite Coulomb repulsion $U$ and the crossing hopping parameter $t^{\prime}$, it turns out that the weights of the Hubbard bands 
are still the same, implying that for large value of $U$ the chemical potential lies in between the two Hubbard bands and the system is Mott insulator. Furthermore, it is noticable that the metallic region is extended as the geometrical frustration reduces the gap between the Hubbard bands. The reduction of the gap implies that the critical correlation-driven metalinsulator transition $U_{c}$ of the frustrated model is larger that that of the unfrustrated one.
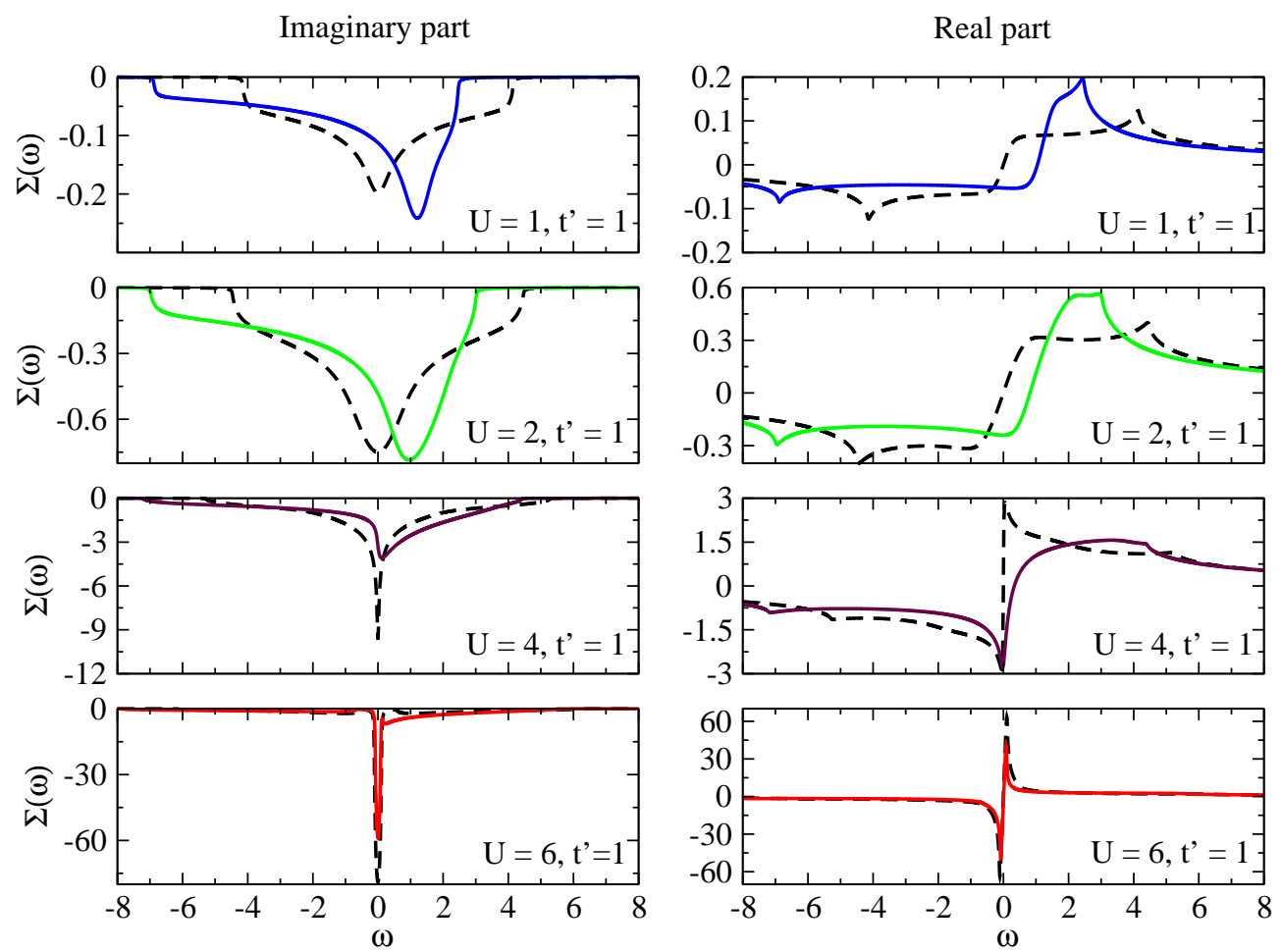

Fig. 4. The imaginary and real part of the self-energy as a function frequency for $t^{\prime}=1$ and different values of $U$. The dashed (solid) lines are of the unfrustrated (strongly frustrated) model, respectively.

Fig. 4 shows the imaginary and real part of the self-energy for the strongly frustrated model $t^{\prime}=1$ with different values of $U$ as chosen in Fig. 3. At Fermi energy, in the insulating regime $U>U_{C}$, the imaginary part of the self-energy has a sharp peak whose weight is roughly independent of $U$. The influence of the geometrical frustration on the critical value $U_{C}$ is presented in Fig. 5 . It turns out that when $t^{\prime}$ is increased, $U_{C}$ is getting larger and finally it levels off at $U_{C} / W\left(t^{\prime}\right) \approx 1.1$ for highly frustrated system. Our prediction $U_{C} / W\left(t^{\prime}\right) \approx 1.1$ when $t^{\prime} \approx 1$ is in good agreement with the single-site DMFT results $[18,19]$. However, that $U_{C}$ calculated within cluster DMFT[13] is approximately twice times larger than that we obtained here. This is not surprised since the importance features of the non-local correlations are not taken into account within single-site theories. 


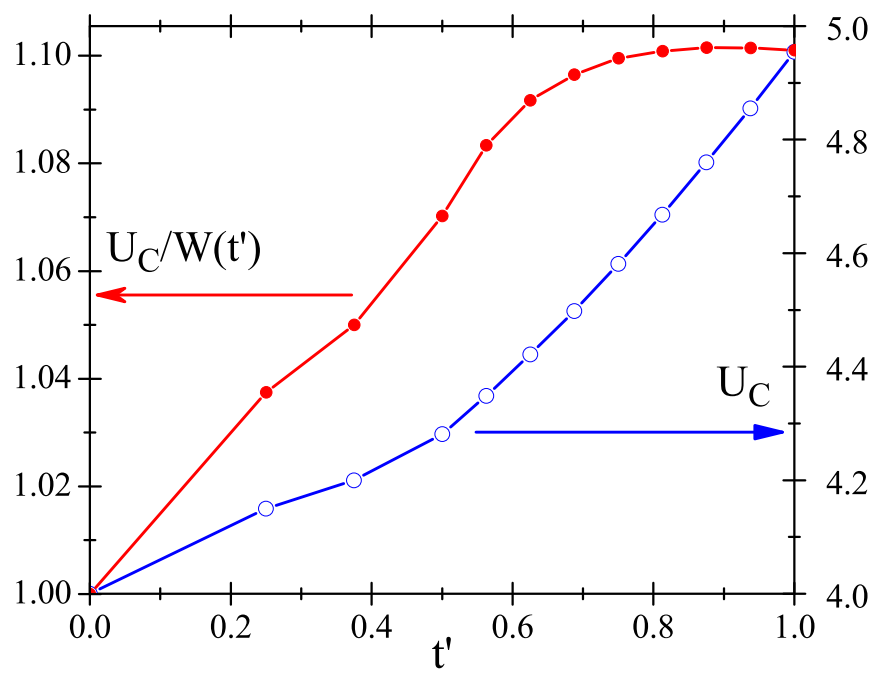

Fig. 5. The critical value $U_{C}$ and $U_{C} / W\left(t^{\prime}\right)$ are shown as a function of the crossing hopping parameter $t^{\prime}$, where $W\left(t^{\prime}\right)$ is the bare bandwidth of the system. Note that $t$ is chosen to be 1 as the energy unit.

\section{CONCLUSIONS}

We have applied the coherent potential approximation to study Mott transition of the half-filled Hubbard model in the two-dimensional square lattice with geometrical frustration. The system has been analyzed for a wide range of the Hubbard on-site Coulomb repulsion $U$ and the crossing hopping integral $t^{\prime}$. It shows that the geometrical frustration reduces the gap between the Hubbard bands. As a result the metallic phase is stabilized up to fairly large Hubbard interactions under the strong geometrical frustration. Our results are in good agreement with the ones obtained by single-site dynamical mean-field theory. However, our $U_{C}$ is approximately half of that obtained by cluster DMFT. This suggests a cluster extention theory of the CPA is needed to get better satisfactory with cluster DMFT results. We leave this problem for future study.

\section{ACKNOWLEDGMENTS}

This research is funded by Vietnam National Foudation for Science and Technology Development (NAFOSTED) under grant number 103.02-2011.05.

\section{REFERENCES}

[1] P. Fulde, J. Phys.: Condens. Matter 16 (2004) S591.

[2] J. S. Gardner, M. J. P. Gingras, and J. E. Greedan, Rev. Mod. Phys. 82 (2010) 53.

[3] S. Kondo et al., Phys. Rev. Lett. 78 (1997) 3729.

[4] D. C. Johnston, J. Low. Temp. Phys. 25 (1976) 145. 
[5] T. Ohashi, T. Momoi, H. Tsunetsugu, and N. Kawakami, Phys. Rev. Lett. 100 (2008) 076402.

[6] N. Bulut, W. Koshibae, and S. Maekawa, Phys. Rev. B68 (2003) 195103.

[7] N. Bulut, W. Koshibae, and S. Maekawa, Phys. Rev. Lett. 95 (2005) 037001.

[8] B. H. Bernhard, B. Canals, and C Lacroix, J. Phys.: Condens. Matter 19 (2007) 145258.

[9] T. Ohashi, T. Momoi, H. Tsunetsugu, N. Kawakami, Prog. Ther. Phys. Suppl. bf 176 (2008) 97.

[10] S. Fujimoto, Phys. Rev. B64 (2001) 085102.

[11] T. Yoshioka, A. Koga, and N. Kawakami, J. Phys. Soc. Jpn. 77 (2008) 104702.

[12] A. Kogaa, T. Yoshiokaa, N. Kawakamia, H. Yokoyamab, Physica C 460 (2007) 1070.

[13] O. Parcollet, G. Biroli, and G. Kotliar, Phys. Rev. Lett. 92 (2004) 226402.

[14] B. Velicky, S. Kirkpatrick, and H. Ehrenreich, Phys. Rev. 175 (1968) 745.

[15] F. Brouers, M. Cyrot, and F. Cyrot-Lackmann, Phys. Rev. B7 (1973) 4370.

[16] F. Ducastelle, J. Phys. C: Solid State Phys. 7 (1974) 1795.

[17] A. Georges, G. Kotliar,W. Krauth, and M. J. Rozenberg, Rev. Mod. Phys. 68 (1996) 13.

[18] M. Rozenberg, R. Chitra, and G. Kotliar, Phys. Rev. Lett 83 (1999) 3498.

[19] T. Ohashi, N. Kawakami, and H. Tsunetsugu, Phys. Rev. Lett 97 (2006) 066401.

Received 27 April 2012. 